\title{
Multimodal Imaging Follow-up of a Thrombosed Developmental Venous Anomaly: CT, CT Angiography and Digital Subtraction Angiography
}

\author{
Kyung Sik Yi, MD', Sang-Hoon Cha, MD', Kyung Soo Min, MD²
}

We report a rare case of thrombosed developmental venous anomaly (DVA) in a 31-year old male with hemorrhagic cerebral venous infarction at the initial clinical presentation. In this case, sequential CT, CT angiography and digital subtraction angiography demonstrated thrombotic obstruction of the venous drainage from DVA, its progressive recanalization and temporal evolution of the affected brain parenchyma. The relevant previous literatures were reviewed and summarized.

Key Words : Developmental venous anomaly; Computed tomography; Digital subtraction angiography; Cerebral venous thrombosis, Cerebral venous infarction

Dilated medullary veins and a prominent draining venous structure constitute a typical developmental venous anomaly (DVA) and it is considered as a benign entity because it barely is associated with any neurological presentations [1]. DVAs associated with arteriovenous shunt causing intracerebral hemorrhages (ICHs) were reported but they are very rare [2-4], and atypical parenchymal type of arteriovenous malformation (AVM) mimicking DVAs were categorized only recently [5]. These clearly suggest DVAs are not a homogenous group of clinical benignancy, rather a

This work was supported by the research grant of the Chungbuk National University in 2010.

Departments of Radiology ${ }^{1}$ and Neurosurgery ${ }^{2}$, Chungbuk National University Hospital, Cheongju, Korea

Received June 7, 2013; accepted after revision June 20, 2013.

Correspondence to: Sang-Hoon Cha, MD, PhD, Department of Radiology, Chung Buk National University Hospital, 1 Circle Rd. 776, Heungduk-gu, Cheongju 361-711, Korea.

Tel. 82.43.269.6473 Fax. 82.43.269.6479

E-mail: shcha@chungbuk.ac.kr

This is an Open Access article distributed under the terms of the Creative Commons Attribution Non-Commercial License (http://creativecommons.org/licenses/by-nc/3.0) which permits unrestricted non-commercial use, distribution, and reproduction in any medium, provided the original work is properly cited. diverse vascular anomaly with various findings.

Thrombosis of a DVA is extremely rare, 21 DVAs with thrombosis in the collecting veins were reported in the previous literatures $[1,6]$. Those cases included 18 supratentorial and three infratentorial cases and showed image findings at initial diagnostic brain imaging mostly. But, serial changes during resolution and recanalization of the thrombosed DVA had not been documented with multimodal image findings.

We present a case of symptomatic DVA presented with hemorrhagic cerebral venous infarction in which repeated multimodal imaging clearly showed temporal changes of the thrombosed collecting vein and the affected brain.

\section{CASE REPORT}

A 31-year-old male presented with loss of consciousness of abrupt onset after several days of headache. Initial pre-contrast $\mathrm{CT}$ showed swollen hypodense area, ICHs and linear hyperdensity in the left frontal lobe (Fig. 1A). CT angiography with 3-dimensional post processing revealed dilated medullary veins with caput medusa appearance, but no venous drainage from the 
left frontal DVA) (Fig. 1B-D). Multiplanar source images of CT angiography demonstrated the filling defect in the anterior superior sagittal venous sinus and suggested the thrombosis in the draining vein (Fig. 1C). On follow-up CT angiograms ten days (not shown in this report) and two months later (Fig. 2B, C), thrombosed draining vein was progressively recanalized and detected were findings of venous infarction with multifocal parenchymal hemorrhages in the left frontal lobe. Repeated digital subtraction angiography two years after the initial clinical presentation (Fig. 2D) clearly showed the presence of resolving venous thrombosis at a month (Fig. 2A).

No underlying cause such as connective tissue disease, coagulopathy, history of steroid administration, dehydration and so on was identified for the spontaneous thrombosis in the venous drainage from DVA.

The patient was treated conservatively, became conscious five days later and eventually recovered without localized neurological deficit. He developed generalized seizure, but had been under control with anti-epileptic medications.

\section{DISCUSSION}

In this case, interpretation of CT and CT angiography findings at the time of initial clinical presentation was complicated because spontaneous ICHs abruptly occurred in a young, healthy male and there was no underlying vascular pathology found such as AVM in the left frontal lobe or dural AVM and the hemorrhages appeared not to be related to neoplasms. Hypodense area in the left lobe involved both cortical gray matter and white matter as in the case of cerebral infarction, did not coincide with the territory of left anterior cerebral artery. Curvilinear hyperdensity coursed from
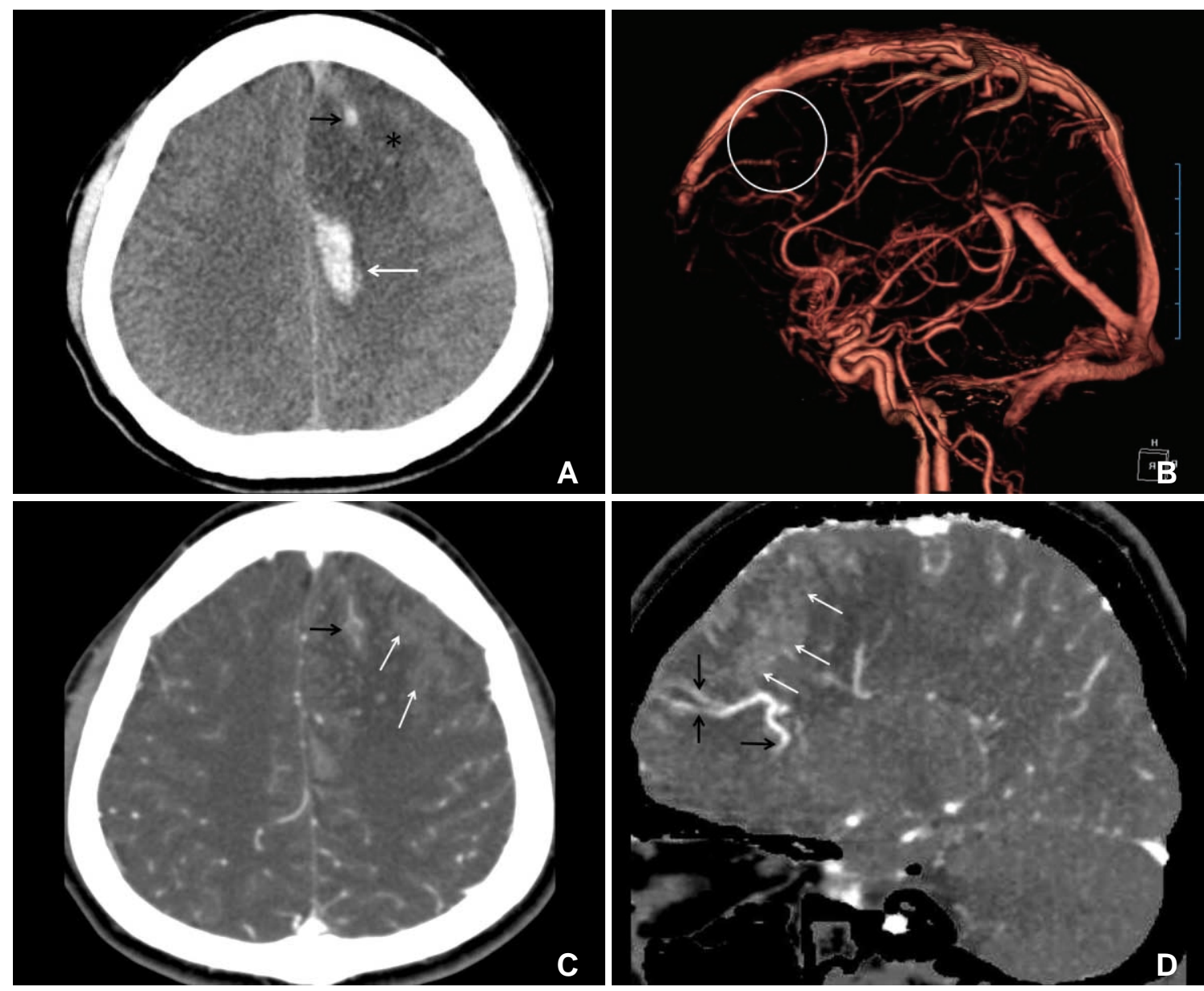

Fig. 1. CT and CT angiography (CTA) images at the day of initial clinical presentation.

Multifocal hyperdense intracerebral hemorrhages (white arrow) and hypodense area (asterisk) are present in the left frontal lobe. Continuing hyperdensity (black arrow) courses from the hypodense area to the superior sagittal sinus (A). 3-dimensionally reconstructed CTA (B) shows no venous drainage to the superior sagittal sinus (white circle). Thrombosed vein (black arrow) and area of parenchymal staining, suggestive of venous congestion (white arrows) is evident on the axial source (C) and sagittal reformatted (D) images, and medullary and collecting veins in the adjacent brain (black arrowheads) are dilated and prominent (D). 
the left frontal parenchyma to the midline anteriorly. Source images from CT angiography showed multiple dilated linear enhancements with parenchymal staining in the left frontal lobe. Possible venous infarction due to thrombosis in the venous drainage was suspected but DVA with thrombosis was not included in the initial differential diagnosis because venous collector was not recognizable. Early correct recognition of thrombosed typical DVA as the underlying cause for the ICH and venous infarction of sudden onset could lead to the proper anticoagulation treatment.

Cases of spontaneous ICH related to DVA were reported and associated with either high flow arteriovenous shunts or cavernous venous malformations [1-4]. However, very small number of typical DVAs were reported, which presented ICH as the initial manifestation [7, 8]. Seki et al. [9] analyzed previously reported 13 cases and their one case of typical DVA complicated by thrombosis of the draining vein. Among those 14 cases, they include one case of venous infarction without ICH by mistake, thus four had ICHs. eight cases of venous infarction including their own two were analyzed by Masson et al [10]. A case of childhood DVA complicated by spontaneous thrombosis was reported elsewhere [11] and should be included in the list of venous infarction. Among those nine, two were categorized as "hemorrhagic" and we reviewed the case reports. Authors of the two case reports related ICHs to thrombosis in the draining veins, and their case figures did not show venous infarction initially $[8,12]$.

Among 21 cases of DVAs with venous thrombosis in the previous literatures, one was reported in French [13] and another one in Spanish [14]. 19 cases reported in English were reviewed [6, 9, 15-18] and we summarized their demographics, location of DVA, presence of parenchymal hemorrhage at the initial
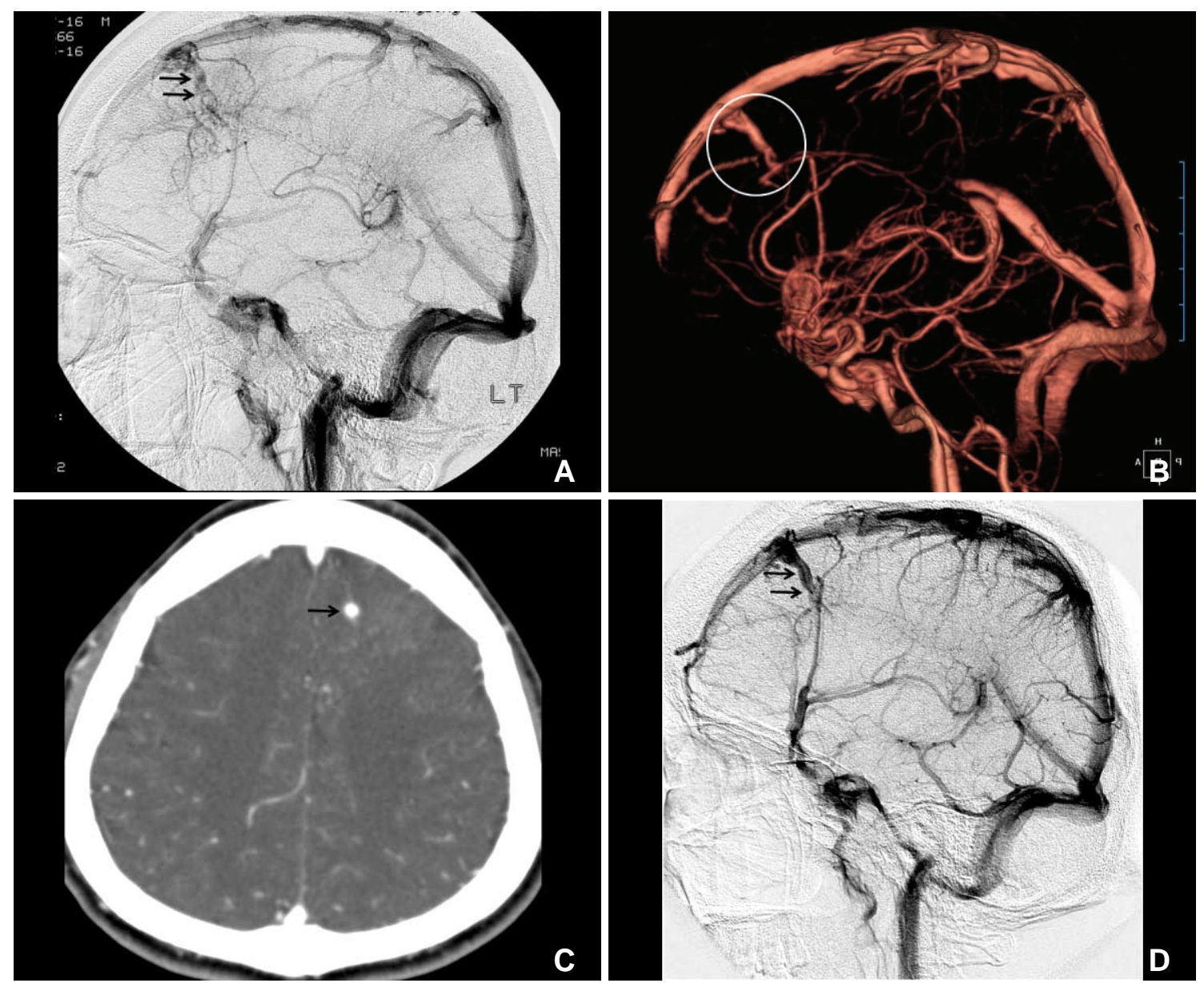

Fig. 2. Follow-up images demonstrating progressive resolution of thrombosis and recanalization of draining vein from the DVA.

DSA at one month shows intravenous filling defects in the left frontal DVA (black arrows), dilated medullary veins, delayed contrast washout and parenchymal staining in the lateral projection image (A). CTA at two month reveals patent draining vein (white circle) on threedimensional reconstruction (B) and axial source image (C) demonstrates corresponding contrast enhancement (black arrow). Note parenchymal staining in the left frontal lobe, evident on the initial CTA (Fig. 1C), is no longer prominent (C). On two- year follow-up DSA, medullary veins are not engorged and draining vein is completely recanalized (D). 
Thrombosed Developmental Venous Anomaly

Table 1. Case Summary of Thrombosed DVAs Previously Reported in English

\begin{tabular}{|c|c|c|c|c|c|}
\hline 1st Author (year*) & $\mathrm{Age}^{\dagger} /$ gender $^{\dagger}$ & DVA location & Venous infarction & ICH on initial CT/MRI & \\
\hline Bouchacourt (1986) & $37 / F$ & $\mathrm{Lt} F$ & $(+)$ & $(-)$ & French $^{\S}$ \\
\hline Yamamoto (1989) & $26 / F$ & Rt P & $(-)$ & $(+)$ & pregnancy \\
\hline Field (1995) & $34 / F$ & Rt T & $(+)$ & $(+)$ & \\
\hline Kim (1996) & $13 / M$ & Rt T & $(+)$ & $(-)$ & mortality \\
\hline Merten (1998) & $50 / F$ & Lt putamen & $(-)$ & $(+)$ & \\
\hline Guerrero (1998) & & Rt cbll & & & Spanish \\
\hline Konan (1999) & $31 / M$ & cbll & $(+)$ & $(-)$ & \\
\hline Lai (1999) & $56 / M$ & Rt P & $(+)$ & $(-)$ & \\
\hline Herbreteau (1999) & $45 / M$ & Lt P & $(+)$ & $(-)$ & \\
\hline Thobois (1999) & $25 / F$ & Rt P & $(+)$ & $(-)$ & \\
\hline Masson (2000) & $43 / M$ & LtP & $(+)$ & $(-)$ & smoker \\
\hline Masson (2000) & $68 / M$ & Lt F & $(+)$ & $(-)$ & \\
\hline Hammoud (2002) & $32 / M$ & Lt F-P & $(+)$ & $(-)$ & \\
\hline Peltier (2004) & $32 / M$ & Lt T-P-O & $(+)$ & $(-)$ & \\
\hline Lovrencic-Huzjan (2004) & 39 / M & cbll & $(-)$ & SAH only & \\
\hline Vieira Santos (2006) & $9 / F$ & Lt F-P & $(+)$ & $(-)$ & \\
\hline Seki (2007) & $33 / \mathrm{M}$ & Lt T-P & $(+)$ & $(+)$ & \\
\hline Gama (2008) & $19 / F$ & Rt F & $(+)$ & $(+)$ & \\
\hline Walsh (2008) & $38 / \mathrm{M}$ & Lt F & $(-)$ & $(-)$ & \\
\hline Walsh (2008) & $52 / M$ & RtP & $(+)$ & $(+)$ & \\
\hline Sepelyak (2010) & $17 / F$ & $\mathrm{Lt} F$ & $(+)$ & $(-)$ & oral contraceptive \\
\hline Present Case (2012) & $31 / M$ & $\mathrm{Lt} F$ & $(+)$ & $(+)$ & \\
\hline
\end{tabular}

* Year of publication of the case report

${ }^{\dagger}$ age in years

${ }^{\dagger} \mathrm{M}$, male; $\mathrm{F}$, female

${ }^{s} \mathrm{~F}$, frontal lobe; T, temporal lobe; P, parietal lobe; F-P, fronto-parietal; T-P-O, temporo-parieto-occipital; cbll, cerebellum

$\S$ main text in French, with English abstract

diagnosis including our case (Table 1). Among those 20, three had cerebellar DVAs [14, 18, 19], and one case presented with only with subarachnoid hemorrhage and no infarction [18]. Two cases had ICH only $[8,12]$ and five cases $[7,9,15,16]$ showed findings of ICH and venous infarction on the initial brain images. Most of them presented only CT, MRI or angiographic images at the initial diagnosis or at certain time points. Only four cases $[7,10,12,20]$ had follow-up images of any sort, but they did not show sequential changes of the thrombosed vein and DVA, neighboring brain tissue.

As described elsewhere, thrombotic obstruction of DVA and draining vein resulting venous congestion should be the pathologic mechanism for the initial parenchymal hemorrhages and venous infarction. In the present case, sequential CT, CT angiography and digital subtraction angiography demonstrated initial thrombotic obstruction of the venous drainage from DVA, its recanalization and temporal changes occurring in the affected brain parenchyma.

Acknowledgement: Editor allowed to extend the number of references.

\section{References}

1. Ruiz DS, Yilmaz H, Gailloud P. Cerebral developmental venous anomalies: current concepts. Ann Neurol 2009;66:271-283 


\section{Kyung Sik Yi, et al.}

2. Roh JE, Cha SH, Lee SY, Lee SY, Jeon MH, Cho BS, et al. Atypical developmental venous anomaly associated with single arteriovenous fistula and intracerebral hemorrhage: a case demonstrated by superselective angiography. Korean J Radiol 2012;13:107-110

3. Oran I, Kiroglu Y, Yurt A, Ozer FD, Acar F, Dalbasti T, et al. Developmental venous anomaly (DVA) with arterial component: a rare cause of intracranial haemorrhage. Neuroradiology 2009;51:25-32

4. Fok KF, Holmin S, Alvarez H, Ozanne A, Krings T, Lasjaunias PL. Spontaneous intracerebral hemorrhage caused by an unusual association of developmental venous anomaly and arteriovenous malformation. Interv Neuroradiol 2006;12:113-121

5. Im SH, Han MH, Kwon BJ, Ahn JY, Jung C, Park SH, et al. Venous-predominant parenchymal arteriovenous malformation: a rare subtype with a venous drainage pattern mimicking developmental venous anomaly. J Neurosurg 2008;108:1142-1147

6. Sepelyak K, Gailloud P, Jordan LC. Thrombosis of a developmental venous anomaly with hemorrhagic venous infarction. Arch Neurol 2010;67:1028

7. Field LR, Russell EJ. Spontaneous hemorrhage from a cerebral venous malformation related to thrombosis of the central draining vein: demonstration with angiography and serial MR. AJNR Am J Neuroradiol 1995;16:1885-1888

8. Yamamoto M, Inagawa T, Kamiya K, Ogasawara H, Monden S, Yano T. Intracerebral hemorrhage due to venous thrombosis in venous agioma: case report. Neurol Med Chir 1989; 29:1044-1046

9. Seki Y, Sahara Y. Spontaneous thrombosis of a venous malformation leading to intracerebral hemorrhage: case report. Neurol Med Chir 2007:47:310-313

10. Masson C, Godefroy O, Leclerc X, Colombani JM, Leys D. Cerebral venous infarction following thrombosis of the draining vein of a venous angioma (developmental abnormality). Cerebrovasc Dis 2000;10:235-238
11. Kim P, Castellani R, Tresser N. Cerebral venous malformation complicated by spontaneous thrombosis. Child Nerv Syst 1996;12: 172-175

12. Merten CL, Knitelius HO, Hedde JP, Assheuer J, Bewermeyer H. Intracerebral haemorrhage from a venous angioma following thrombosis of a draining vein. Neuroradiology 1998;40:15-18

13. Bouchacourt E, Carpena JP, Bories J, Koussa A, Chiras J. Ischemic accident caused by thrombosis of a venous angioma. Apropos of a case. J Radiol 1986;67:631-635

14. Guerrero AL, Blanco A, Arcaya J, Cach J. Venous infarct as presenting form of venous angioma of the posterior fossa. Rev Clin Esp 1998;198:484-485

15. Walsh M, Parmar H, Mukherji SK, Mamourian A. Developmental venous anomaly with symptomatic thrombosis of the draining vein. J Neurosurg 2008;109:1119-1122

16. Gama RL, Nakayama M, Tavora DG, Bomfim RC, Carneiro TC, Pimentel LH. Thrombosed developmental venous anomaly associated with cerebral venous infarct. Arq Neuropsiquiatr 2008;66: 560-562

17. Vieira Santos A, Saraiva P. Spontaneous isolated non-haemorrhagic thrombosis in a child with developmental venous anomaly: case report and review of the literature. Childs Nerv Syst 2006;22:1631-1633

18. Lovrencic-Huzjan A, Rumboldt Z, Marotti M, Demarin V. Subarachnoid haemorrhage headache from a developmental venous anomaly. Cephalalgia 2004;24:763-766

19. Konan AV, Raymond J, Bourgouin P, Lesage J, Milot G, Roy D. Cerebellar infarct caused by spontaneous thrombosis of a developmental venous anomaly of the posterior fossa. AJNR Am J Neuroradiol 1999;20:256-258

20. Peltier J, Toussaint P, Desenclos C, Le Gars D, Deramond H. Cerebral venous angioma of the pons complicated by nonhemorrhagic infarction. case report. J Neurosurg 2004;101:690-693 